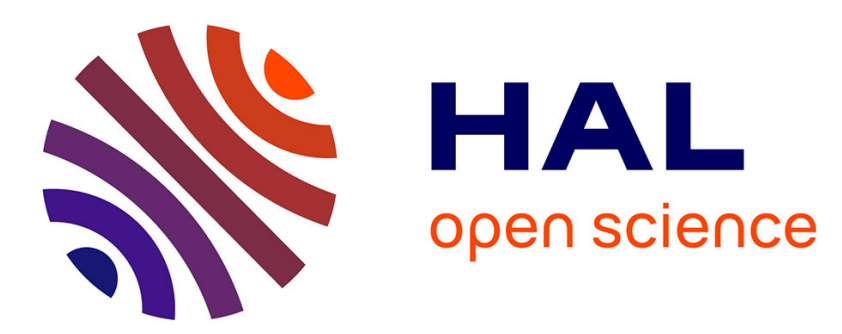

\title{
A Hexapod Walking Robot Mimicking Navigation Strategies of Desert Ants Cataglyphis
}

\author{
Julien Dupeyroux, Julien Serres, Stéphane Viollet
}

\section{To cite this version:}

Julien Dupeyroux, Julien Serres, Stéphane Viollet. A Hexapod Walking Robot Mimicking Navigation Strategies of Desert Ants Cataglyphis. Biomimetic and Biohybrid Systems, pp.145-156, 2018, 978-3319-95972-6. 10.1007/978-3-319-95972-6_16 . hal-02075674

\section{HAL Id: hal-02075674 \\ https://hal-amu.archives-ouvertes.fr/hal-02075674}

Submitted on 21 Mar 2019

HAL is a multi-disciplinary open access archive for the deposit and dissemination of scientific research documents, whether they are published or not. The documents may come from teaching and research institutions in France or abroad, or from public or private research centers.
L'archive ouverte pluridisciplinaire HAL, est destinée au dépôt et à la diffusion de documents scientifiques de niveau recherche, publiés ou non, émanant des établissements d'enseignement et de recherche français ou étrangers, des laboratoires publics ou privés. 


\title{
AntBot, a hexapod walking robot mimicking navigation strategies of desert ants Cataglyphis
}

\author{
Julien Dupeyroux, Julien Serres, and Stéphane Viollet \\ Aix Marseille Univ., CNRS, ISM UMR 7287, Marseille, France \\ stephane.viollet@univ-amu.fr, \\ http://www.biorobotics.eu/
}

\begin{abstract}
In this study, a desert ant-inspired celestial compass and a bio-inspired minimalist optic flow sensor named $\mathrm{M}^{2} \mathrm{APix}$ (which stands for Michaelis Menten Auto-adaptive Pixels), were embedded onboard our $2 \mathrm{~kg}$-hexapod walking robot called AntBot, in order to reproduce the homing behavior observed in desert ants Cataglyphis fortis. The robotic challenge here was to make the robot come back home autonomously after being displaced from its initial location. The navigation toolkit of AntBot comprises the celestial-based heading direction, and both strideand ventral optic flow-based odometry, as observed in desert ants. Experimental results show that our bio-inspired approach can be useful for autonomous outdoor navigation robotics in case of GPS or magnetometer failure, but also to compensate for a drift of the inertial measurement unit. In addition, our strategy requires few computational resources due to the small number of pixels (only 14 here), and a high robustness and precision (mean error of $4.8 \mathrm{~cm}$ for an overall path ranging from $2 \mathrm{~m}$ to $5 \mathrm{~m})$. Finally, this work presents highly interesting field results of antbased theoretical models for homing tasks that have not been tested yet in insectoid robots.
\end{abstract}

Keywords: Celestial compass, Polarized light, Optic flow, Outdoor navigation, Homing, Odometry, Path integration, Legged robot, Biorobotics

\section{Introduction}

Most insects, especially desert ants Cataglyphis, are experts in daily long-range navigation, reaching highly robust precision in locating significant areas (nest, food). Due to the extreme heat, desert ants cannot use pheromones to track their navigating path. However, they are equipped with a useful navigation toolkit comprising: (i) a path integration (PI) routine relying on celestial cues, and both stride and ventral optic flow integration, and (ii) a view-based landmark guidance where panoramic snapshots are memorized to retrieve and follow routes established in cluttered environments [1-3]. It has been shown that desert ants keep their PI updated whenever they follow familiar routes or not. However, as PI is prone to accumulative errors, desert ants will opt for landmark-based navigation when visual cues are available [4]. 
The sensory modalities involved in desert ants Cataglyphis PI strategy are combined to compute a homing vector, namely a vector (distance and heading direction) constantly pointing toward the nest when foraging. The heading direction information is computed based on celestial cues: the sun position in the sky and the direction of linearly polarized skylight (e-vectors) in the zenith part of the sky [5]. The acquisition of the polarized cues is found in the insect's dorsal rim area (DRA) where photoreceptors are sensitive to the direction of polarization [6], mostly in the ultraviolet (UV) range [7]. Then, desert ants estimate their distance from both stride [8] and ventral optic flow [9] integration, though Cataglyphis are known to correct the estimated distance in the absence of any optic flow information.

Former implementations of the desert ants navigational toolkit have led to very interesting results. The Sahabot 1 and 2 projects $[10,11]$ experimented celestial compass on board wheeled robots in an ant-like homing navigation task with average error of $13.5 \mathrm{~cm}$. The sensor was composed of three polarization units, each of them combining two visible polarized light sensors with orthogonal polarization selectivity. According to the Labhart's polarization opponent model [6], they computed the direction of polarization of the moving robot. More recently, a miniaturization of this celestial compass was proposed by $[12,13]$, embedded onboard a small rover in [14] and tested in ant-like homing navigation tasks. The average error in these experiments was equal to $42 \mathrm{~cm}$, and their odometer used wheel encoders. Interesting investigations have been conducted on polarization vision $[15,16]$. Yet, it seems no other full robotic implementation of polarization vision has been used as an input for autonomous outdoor navigation tasks.

In this paper, we propose to test our ant-inspired 2-pixel UV-polarized light celestial compass and our 12-pixels $\mathrm{M}^{2} \mathrm{APix}$ bio-inspired ventral optic flow sensor on board our hexapod walking robot called AntBot. The challenge was to make the robot autonomously come back to its initial location after being randomly displaced, with outbound trajectories ranging from $4 \mathrm{~m}$ to $8 \mathrm{~m}$. The AntBot insectoid robot is fully discribed in section 2 . The homing procedure is outlined in section 3, and field results are displayed and discussed in section 4.

\section{AntBot, the robotic ant}

\subsection{Designing the hexapod walking robot}

AntBot is a six-legged walking robot designed to mimic desert ants Cataglyphis fortis (Fig. 1), first on the morphological and locomotive aspects, then on the sensory modalities and navigation skills. Each leg has three joints actuated by means of Dynamixel AX 18 servomotors, integrated in a fully 3D-printed structure (printing being made with polyactic acid (PLA) filament). The servos are all connected to an Arduino-like micro-controller (the OpenCM 9.04C board) through USART serial communication. An extra degree of freedom has been added to control the roll of the robot while walking, but in this study the roll actuation is only used for the heading estimation. The locomotion firmware of AntBot has been adapted from the one used for his predecessor Hexabot [17] 
and thus benefits from high walking stability. AntBot is mastered by a Raspberry $\mathrm{Pi} 2 \mathrm{~B}$ micro-computer, which communicates with all sensors as described in the robot's electronic architecture shown in figure 1. A WiFi communication can be established between the robot's computer unit and the host computer. The robot is powered by a three cells $11.4 \mathrm{~V} 5300 \mathrm{mAh}$ lithium polymer battery (Gens ACE), with a maximum autonomy of 30 minutes.
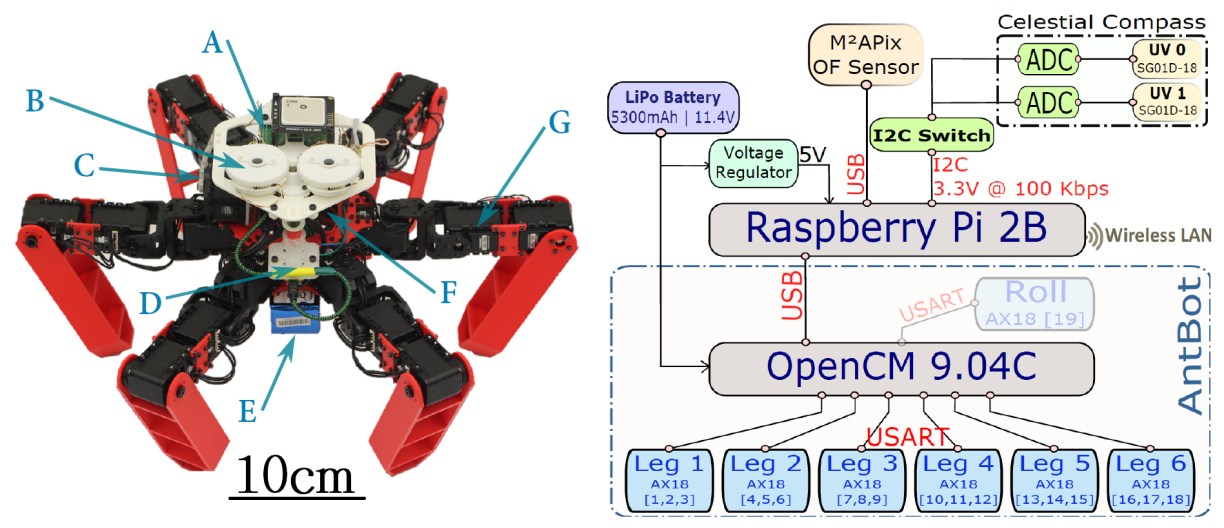

Fig. 1. Left: Photography of AntBot. (A) AntBots micro-computer Raspberry Pi 2B placed below its top shelf (in white). (B) The celestial compass with its two POL-units $U V_{0}$ and $U V_{1}$ looking at the zenith part of the sky dome. (C) Roll actuation of the top shelf. (D) Ventral optic flow sensor called $\mathrm{M}^{2}$ APix. (E) AntBot's powering battery (Gens ACE, 11.4V 5300mAh). (F) AntBot's micro-controller OpenCM 9.04C, set on top of the battery. (G) Dynamixel AX18 servomotors. Right: Hardware architecture of AntBot. The robot's low-level electronics, including the micro-controller ant the 19 servomotors, are gathered within the dashed line.

\subsection{The celestial compass}

To compute its heading direction while navigating, AntBot makes use of its antinspired celestial compass embedded on its roll-actuated shelf. It is composed of two UV-light photodiodes SG01D18 (SgLux) topped with rotating linear sheet polarizers (HNP'B replacement), for a final spectral sensitivity from $270 \mathrm{~nm}$ to $400 \mathrm{~nm}$ with peak transmission at 330nm (Fig. 2). Each polarization unit (POLunit), namely $U V_{0}$ and $U V_{1}$, has a refreshing rate of $33 \mathrm{~Hz}$ and an angular field of view of approximately $100^{\circ}$. Former investigations showed that our celestial compass successfully worked under various weather conditions, even with very poor UV-index $[18,19]$.

Let $x$ be the orientation of the linear sheet polarizers. According to the polarization pattern of the skylight at the zenith, $U V_{0}(x)$ and $U V_{1}(x)$ are expected to be $\pi$-periodic sine waves (see [18] for details). Consequently, these raw signals 

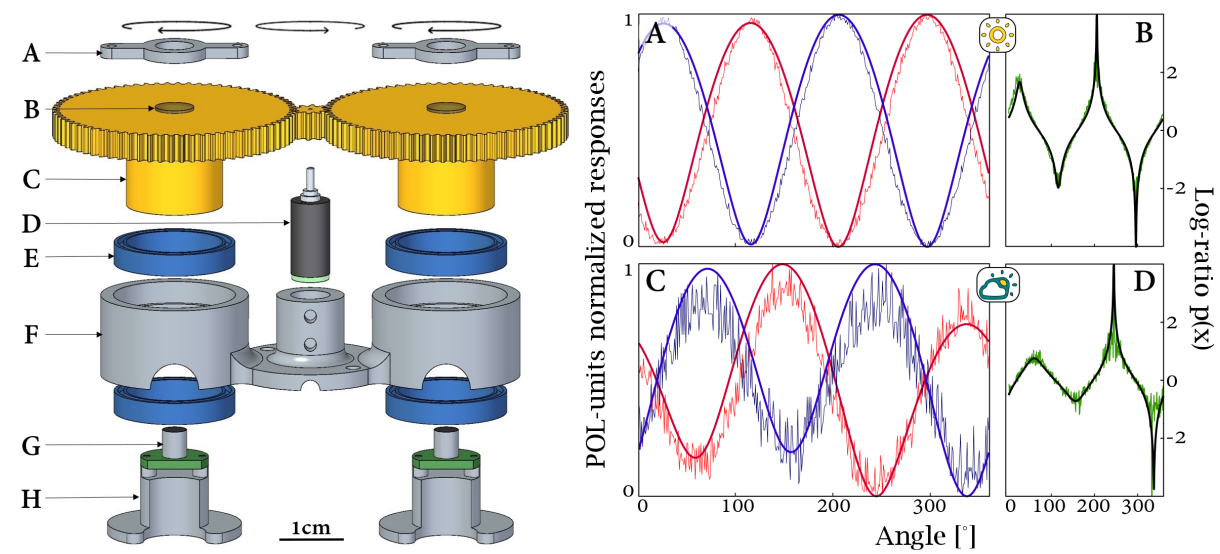

Fig. 2. Left: Exploded view of the celestial compass. (A) Fixation for the UV sheet polarizers (B), holded by rotating gears (C). (D) Stepper motor AM0820-A-0,2257 (Faulhaber). (E) Ball bearings. (F) Celestial compass frame. (G) UV-light sensors SG01D-18 (SgLux) mounted on supports (H). Right: Examples of signals. Graphs (A,C) display normalized raw and corrected outputs of the celestial compass ( $U V_{0}$ in red, $U V_{1}$ in blue), and graphs (B,D) display the corresponding raw (green) and corrected (black) log-ratio signals involved in the computation of the robot's heading direction. Data were collected in Marseille in April, 2017, under both clear (A,B) and cloudy (C,D) weather conditions (UV-index equal to 7).

are first low-pass filtered and then normalized between $\epsilon$ and 1 as described in figure $2\left(\epsilon \sim 10^{-6}\right.$ is set to prevent from logarithm computation failure). We then compute the log-ratio $p(x)$ of the two normalized and corrected signals $U V_{0}^{n c}(x)$ and $U V_{1}^{n c}(x)$, therefore:

$$
p(x)=\log _{10}\left(\frac{U V_{1}^{n c}(x)}{U V_{0}^{n c}(x)}\right)
$$

In a $[0 ; \pi]$ interval, the angle of polarization corresponds to the fiber angle for the maximum value of the $p$-function, while the angle direction $\Psi$ of the solar meridian is depicted by the fiber angle for the minimum value. At this stage of the heading direction computation, $\Psi$ is considered between $0^{\circ}$ and $180^{\circ}$. Consequently, we have:

$$
\Psi=\frac{1}{4}(\underset{x \in[0 ; \pi]}{\arg \min } p(x)+\underset{x \in[0 ; \pi]}{\arg \max } p(x)+\underset{x \in[\pi ; 2 \pi]}{\arg \min } p(x)+\underset{x \in[\pi ; 2 \pi]}{\arg \max } p(x)-\pi)
$$

Due to the physical properties of the Rayleigh's scattering of sunlight, it is not possible to determine the absolute orientation of AntBot from the polarized light celestial compass. In their project Sahabot, Lambrinos et al. used 8 photodiodes to detect the angular sector corresponding to the highest illumination (e.g. the sun location) [10]. Here, the solar/anti-solar ambiguity is treated as follows: the 
top shelf is rolled left and right to get the sun position which corresponds to the highest UV level measured by the two POL-units. The final decision is taken based on the algorithm depicted by Fig. 3 on the basis of the initial heading direction measured $\Psi_{I N I T}$ when the robot is placed with the sun exactly aligned with its longitudinal axis.
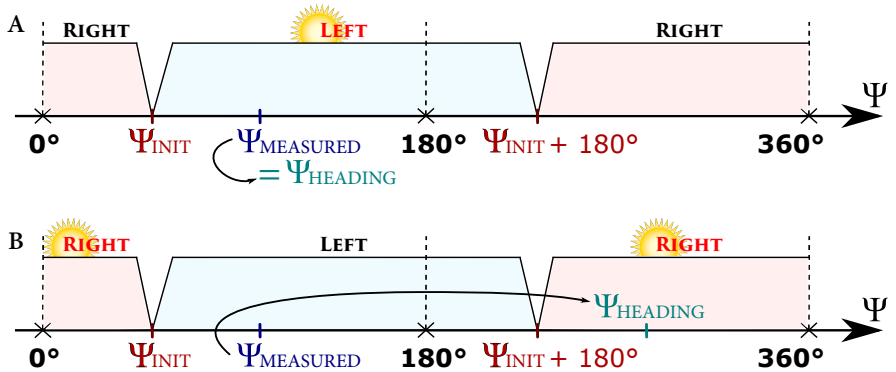

Fig. 3. Principle of solar-based ambiguity resolution of the heading direction computation. (A) The measured heading direction $\Psi_{M E A S U R E D}$ is located in the LEFT angular sector as the sun is located on the left of the robot. Consequently, $\Psi_{H E A D I N G}=$ $\Psi_{M E A S U R E D}$. (B) In this case, the measured heading direction is still located in the LEFT angular sector but the robot detects the sun on its right: $\Psi_{H E A D I N G}=$ $\Psi_{M E A S U R E D}+180^{\circ}$.

When the rolling procedure leads to very similar left and right UV-levels, then the robot decides whether $\Psi=\Psi_{I N I T}$ or $\Psi=\Psi_{I N I T}+180^{\circ}$ just by integrating its stride-based orientation. Indeed, despite this estimation is poor due to cumulative drift, it is good enough to be used as a cue for disambiguation. Last, the sun deviation was corrected using a solar ephemeris table with respect to the time and location of each experiments.

\subsection{The ventral optic flow sensor}

Our hexapod AntBot also integrates a 12-pixel ventral optic flow sensor called $\mathrm{M}^{2}$ APix (Michaelis-Menten Auto-adaptive Pixels, Fig. 4, [20]) which main advantage consists in auto-adaptability in a 7-decade light range, with appropriate responses when measuring signals that change up to \pm 3 decades. This attribute makes $\mathrm{M}^{2} \mathrm{APix}$ suitable for outdoor experiments where light variations occur randomly.

The ventral optic flow $\omega($ in $\mathrm{rad} / \mathrm{s})$ is defined as follows:

$$
\omega=\frac{\Delta \varphi}{\Delta T}=\frac{\mathcal{V}}{D}
$$

with $\Delta \varphi$ the inter-pixel angle between two adjacent pixels in a row (Fig. 4), $\Delta T$ the time delay measured between two adjacent pixels, $\mathcal{V}$ the robot's speed and $D$ the height-to-the-ground of the $\mathrm{M}^{2} \mathrm{APix}$ sensor. Former characterization 
of the sensor used in AntBot showed that $\Delta \varphi$ is equal to $3.57^{\circ}$ with standard deviation of $0.027^{\circ}$ (see [21] for details). $\Delta T$ is computed using the cross-correlation method described in [22]. The height variation of AntBot does not exceed $1 \mathrm{~cm}$ while walking. On average, these small variations do not disturb $\mathrm{M}^{2} \mathrm{APix}$ measurements enough to cause navigation failure. In particular, this property is guaranteed by a threshold process ahead the cross-correlation computation.

A

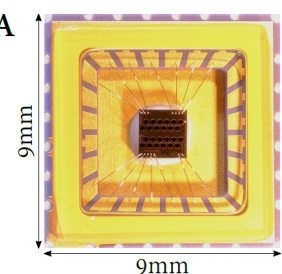

B

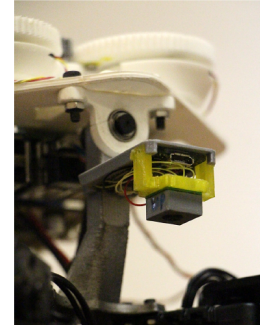

C

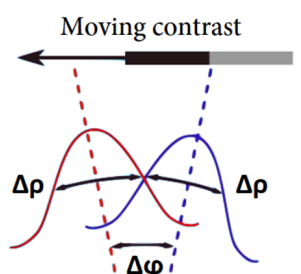

Defocused

i

i, lens

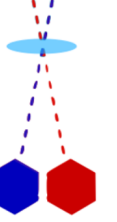

Pixel 1 Pixel 2
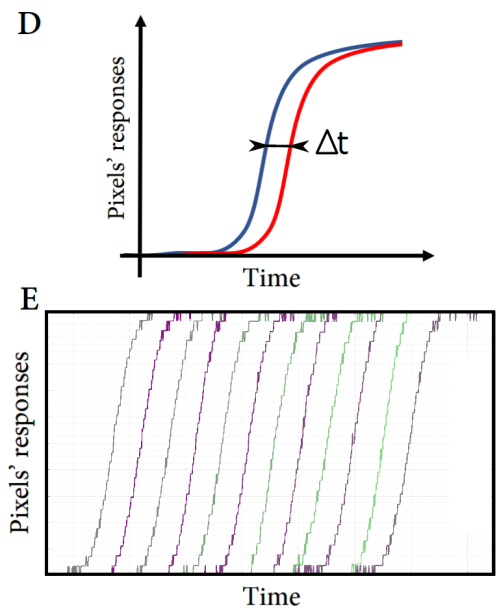

Fig. 4. (A) The $\mathrm{M}^{2}$ APix silicon retina. Adapted from [20]. (B) Photography of the $\mathrm{M}^{2} \mathrm{APix}$ sensor topped with the optics of a Raspberry Pi NoIR Camera and connected to the Teensy 3.2 micro-controller. (C) Optics geometry explaining how the optic flow detection is operated. $\Delta \varphi$ is the inter-pixel angle between two adjacent pixels forming a local motion sensor (LMS). $\Delta \rho$ is the acceptance angle given by the width of the Gaussian angular sensitivity at half height. Adapted from [22]. (D) Theoretical signals obtained for pixels 1 and 2 from (C) according to the moving contrast. Adapted from [22]. $\Delta T$ is the time delay between the two pixels and is used for optic flow computation. (E) Real signals obtained for the 12 pixels when detecting a moving edge.

\section{The ant-inspired navigation model}

Let $\Psi_{R O B O T}$ be the orientation of the robot relative to the ground horizontal $\mathrm{X}$-axis, $\Psi_{C O M P}$ its orientation according to the solar azimuth obtained with the celestial compass, $\Psi_{I N I T}$ the initial orientation given by the celestial compass, and $\Psi_{R E L E A S E}$ the orientation of the robot after being released on the ground, also given by the celestial compass. Every angle value is given in degrees. The location of the robot is given for each homing checkpoint $C_{i}$ by its $(X[i], Y[i])$ position. The initial position is set at $(0,0)$ and the release position is provided by the operator and denoted as $\left(X_{\text {release }}, Y_{\text {release }}\right)$. All Cartesian coordinates are given in centimeters. When the robot is released on a random place on 
the ground, the homing distance Dist ${ }_{H O M I N G}$ and orientation $\Psi_{H O M I N G}$ are computed using equations 4 and 5 respectively. The combination of the homing distance and direction forms the robot's homing vector, as described in desert ants.

$$
\begin{gathered}
\text { Dist }_{\text {HOMING }}=\sqrt{X_{\text {release }}^{2}+Y_{\text {release }}^{2}} \\
\Psi_{H O M I N G}= \begin{cases}\operatorname{atan}\left(\frac{Y_{\text {release }}}{X_{\text {release }}}\right), & \text { if } X_{\text {release }}<0 \\
180+\text { atan }\left(\frac{Y_{\text {release }}}{X_{\text {release }}}\right), & \text { if } X_{\text {release }}>0\end{cases}
\end{gathered}
$$

In case the position along the $\mathrm{X}$-axis is equal to 0.00 (with float precision), the heading direction is chosen between $0^{\circ}$ and $180^{\circ}$ on the basis of turning stride integration. The homing rotation order to be applied $R_{H}$ is given by:

$$
R_{H}=\Psi_{H O M I N G}-\Psi_{R E L E A S E}
$$

The stride order is computed as the Euclid division of Dist ${ }_{H O M I N G}$ by the average stride length $d_{\text {Stride }}$, then equally split into the $N_{H}$ homing checkpoints. For each checkpoint $C_{i}, i \in[1 . .10]$, the current orientation of the robot $\Psi_{R O B O T}[i]$ is computed as follows:

$$
\Psi_{R O B O T}[i]=\Psi_{C O M P}[i]-\Psi_{I N I T}
$$

The walked distance Dist $[i]$, estimated from the robot's sensors and stride, from checkpoint $C_{i-1}$ to checkpoint $C_{i}$ is computed as the mean between the static estimate of distance provided by the stride integrator, and the dynamic estimate of distance provided by the ventral optic flow sensor as given in eq.3:

$$
\operatorname{Dist}[i]=\frac{1}{2}\left(\operatorname{Stride}[i] \cdot d_{\text {Stride }}+\beta \cdot \frac{D \cdot \Delta \varphi \cdot T_{S T R I D E}[i]}{\Delta T[i]}\right)
$$

where Stride $[i]$ is the number of strides executed, $\beta$ is an empiric gain, $D$ is the distance to the ground of the $\mathrm{M}^{2} \mathrm{APix}$ sensor, $\Delta \varphi$ is the inter-pixel angle of the $\mathrm{M}^{2} \mathrm{APix}, T_{S T R I D E}[i]$ is the walking time, and $\Delta T[i]$ is the time delay between two adjacent pixels which detect the same light variation. The robot then computes its current location $(X[i], Y[i])$ relative to its release point:

$$
\left\{\begin{array}{l}
X[i]=X[i-1]+\operatorname{Dist}[i] \cdot \cos \left(\Psi_{\text {RОвот }}[i]\right) \\
Y[i]=Y[i-1]+\operatorname{Dist}[i] \cdot \sin \left(\Psi_{\text {RОВот }}[i]\right)
\end{array}\right.
$$

The homing procedure is divided into $N_{H}$ checkpoints separated by steady distances. For each checkpoint $C_{i}, i \in\left[1 . . N_{H}\right]$, AntBot acquires its new heading, and computes a new homing angle (Eq. 5 , using the new $(X[i], Y[i])$ coordinates) which is compared to the current one. If the two homing angles differs by more than one turning stride, then AntBot updates its homing vector. The same test is made on the homing distance (using Eq. 4 with the correct coordinates). These 
$N_{H}$ homeward checkpoints therefore make the robot able to precisely estimate its drift and correct its ballistic trajectory toward the goal location.

Choice of the parameter $\beta$ in Eq. 8. The Dynamixel servos used in AntBot exhibit varying dynamic behavior in accordance with the ambient temperature. Consequently, the estimated average distance traveled may differ between morning and afternoon experiments. Besides, the first and last strides are prone to highly variable length as the robot is stepping from null to maximum speed, and vice versa, which involves high optic flow measurements. Therefore, if the number of strides to be applied is low, the optic flow disturbances will inevitably cause wrong distance estimate. To solve these issues, a set of empiric gains $\beta$ has been used to correct the optic flow measurements (Table 1).

Table 1. Empiric gain $\beta$ used for the outdoor experiments. $\beta_{M}$ stands for the morning value of $\beta$, and $\beta_{A}$ is for the afternoon value.

\begin{tabular}{rcl}
\hline Number of strides & $\beta_{M}$ & $\beta_{A}$ \\
\hline 1 or 2 & 0.667 & 0.500 \\
3 & 0.850 & 0.750 \\
More than 3 & 0.980 & 0.980 \\
\hline
\end{tabular}

\section{Experimental Results}

According to its firmware, several parameters can be adjusted in order to set AntBot's walking tripod gait (Table 2). The values used for the experiments led to the following gait characteristics: AntBot's straight forward walking speed was approximately $10 \mathrm{~cm} / \mathrm{s}$ with an average stride length $d_{\text {Stride }}$ equal to $8.2 \mathrm{~cm}$, and its average turning angle per turning stride is equal to $10.9^{\circ}$. These characteristics highly depend on the environmental conditions, especially in terms of temperature. Finally, the height of the $\mathrm{M}^{2} \mathrm{APix}$ sensor $D$ in the experimental conditions is constant and equal to $17 \mathrm{~cm}$.

Each experiment is organized as follows: the operator first places the robot onto its departure location $(0,0)$. AntBot then computes its initial heading angle $\Psi_{I N I T}$, before being displaced to a random location $\left(X_{\text {release }}, Y_{\text {release }}\right)$ with a random heading angle $\Psi_{R E L E A S E}$. This angle is acquired by AntBot before computing its homing vector. Then the homing procedure is executed until AntBot reaches its goal with a distance error less than one stride length $(i e 8.2 \mathrm{~cm})$. The number of checkpoints $N_{H}$ was set at 8 .

The experiments were performed in Marseille, south of France $\left(43^{\circ} 14^{\prime} 02.1^{\prime \prime}\right.$ N, 526'37.4" E), from February 15 to February 25, 2018, under clear sky conditions without any day-time preference. According to the European Space Agency 
Table 2. Description of the walking gait parameters of AntBot's firmware.

\begin{tabular}{|c|c|c|c|c|}
\hline Parameters & Description & Min & $\operatorname{Max}$ & AntBot \\
\hline FREQ & $\begin{array}{l}\text { Frequency of execution of the } \\
\text { walking strides. }\end{array}$ & $0.2 \mathrm{~Hz}$ & $3 \mathrm{~Hz}$ & $1.0 \mathrm{~Hz}$ \\
\hline DX & $\begin{array}{l}\text { Amplitude of a straight for- } \\
\text { ward stride length. }\end{array}$ & - & - & $8.2 \mathrm{~cm}$ \\
\hline TURN & Amplitude of a turning stride. & - & - & $10.9^{\circ}$ \\
\hline ALT & $\begin{array}{l}\text { Height of the legs' end during } \\
\text { the transfer phase. }\end{array}$ & $10 \mathrm{~mm}$ & $50 \mathrm{~mm}$ & $20 \mathrm{~mm}$ \\
\hline $\mathrm{H}$ & $\begin{array}{l}\text { Height of the robot's center of } \\
\text { mass. }\end{array}$ & $55 \mathrm{~mm}$ & $145 \mathrm{~mm}$ & $75 \mathrm{~mm}$ \\
\hline
\end{tabular}

(ESA), the UV-index was slightly varying around 1.6 (index given under clear sky). Five experiments were conducted, each of them corresponding to a unique and random release location. To show how precise and robust this ant-inspired strategy is, the same experiments were conducted without using any sensor: the homing vector was computed and updated only on the basis of the stride integrator. Consequently, this blind approach prevents AntBot from getting its angular and distance drifts along its inbound trajectory.

The results are displayed in Fig. 5. When using the blind method, AntBot resulted in an mean position error equal to $124 \mathrm{~cm}$ with high variability (sd: $59 \mathrm{~cm}$ ). When homing with the celestial compass and the ventral optic flow sensor, AntBot drastically reduced its error: the average homing error is equal to $4.8 \mathrm{~cm}$ with low variability $(\mathrm{sd}: 1.8 \mathrm{~cm})$. Besides, whether using the blind or the full sensor method, AntBot always stops when it considers to be less than one stride away from its goal. In the particular case where homing is performed with the ant-inspired sensors, AntBot actually stops in this area, even if its real location slightly differs from where it believes to be: the average distance error between real and believed locations is $5.4 \mathrm{~cm}$, which jumps to $120 \mathrm{~cm}$ with the blind homing procedure.

\section{Conclusion}

We designed a new ant-inspired hexapod robot called AntBot and tested in real homing navigation experiments. AntBot is ant-like at every level of its conception: first, its overall structure is designed after the ants' thorax and legs (6 legs, hexagonal shape); the poor resolution of the insect vision is also reproduced with only 14 pixels (the vision of polarization, as observed in the DRA, and the perception of ventral optic flow); last, the path integration navigation behavior of desert ants Cataglyphis has been reproduced with remarkable results.

AntBot is an outstanding example of what biorobotics means: this is a fully autonomous navigating robot that does not suffer from the limitations of conventional tools (low-resolution GPS, IMUs' drift, and the high computational 


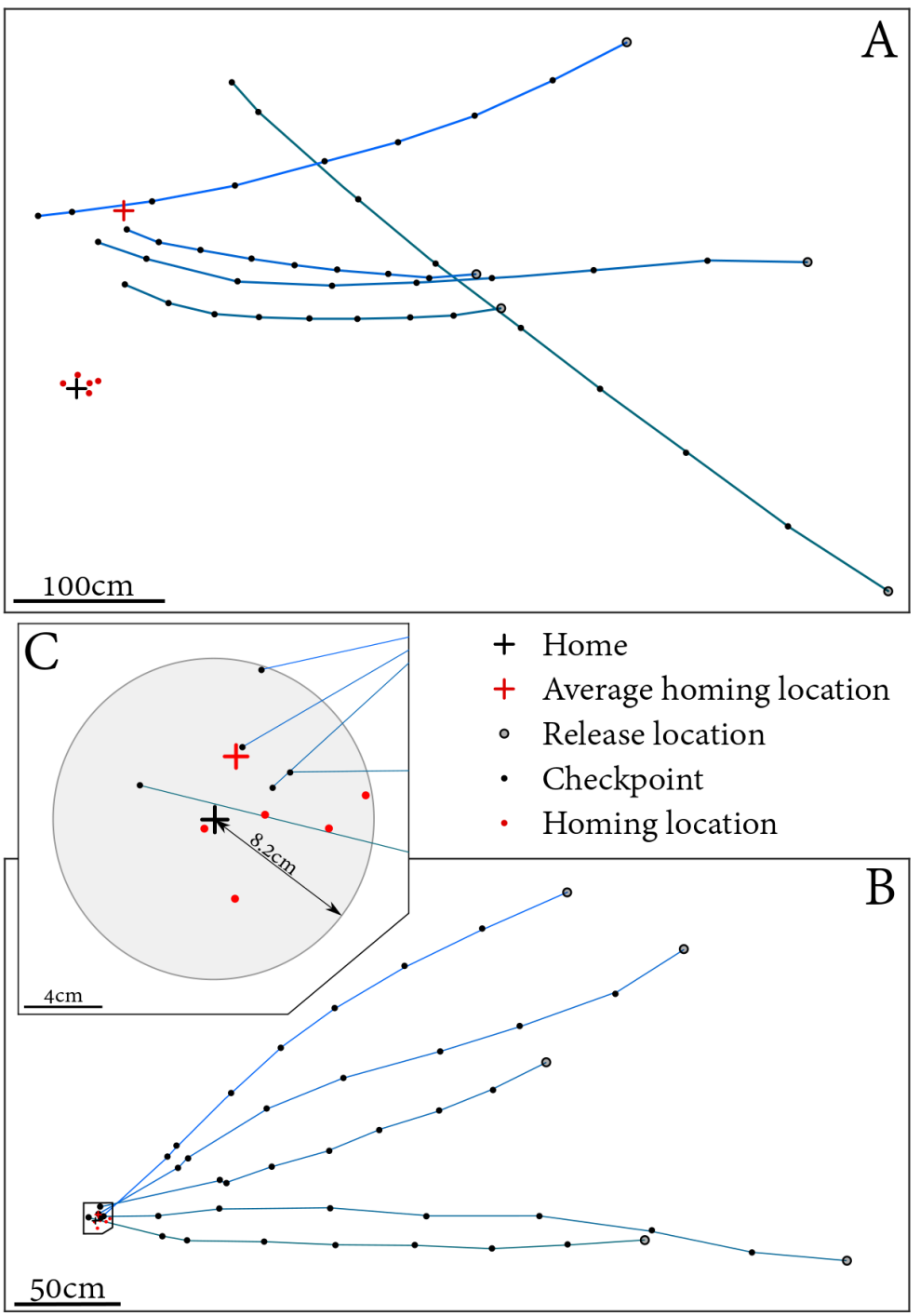

Fig. 5. Overall results of the homing experiments. Black cross: initial location to be reached. Red cross: average final position. Red dots: where the robot believes to be at the end of the homing procedure. Black circles: release locations. (A) Homing trajectories according to the blind method (both distance and orientation are computed by stride integration). (B) Homing trajectories when the robot uses the celestial compass to compute its heading direction, and merges both ventral optic flow and stride integration to estimate its travel distance. (C) Magnified view of the homing results in (B); the circle depicts the positions that are less than one stride away from the goal. 
cost of SLAM methods, for example), and we hope it will give rise to some interesting discussions among the members of the biologists' community, on topics such as navigation. In that sense, AntBot could be considered in testing neural models of the insects' path integrator like those proposed in [23, 24].

Future work will focus on the robustness and precision of the presented method with respect to variable meteorological conditions, and will be compared to gradual integration of the sensors in the path integration model. The odometry will also be investigated to reduce the stride-based distance estimation error [25]. Soon, AntBot will be asked to travel random trajectories before coming back to its departure location.

\section{Acknowledgments}

The authors would like to thank Marc Boyron and Julien Diperi for their technical support in the conception of the celestial compass.

\section{Funding}

This work was supported by the French Direction Générale de l'Armement (DGA), CNRS, Aix-Marseille University, the Provence-Alpes-Côte d'Azur region, and the French National Research Agency for Research (ANR) with the Equipex/Robotex project.

\section{References}

1. Muller, M., Wehner, R.: Path integration in desert ants, Cataglyphis fortis. In: Proceedings of the National Academy of Sciences, 85(14), 5287-5290 (1988).

2. Collett, M., Collett, T. S., Bisch, S., Wehner, R.: Local and global vectors in desert ant navigation. In: Nature, 394(6690), 269 (1998).

3. Wehner, R.: Desert ant navigation: how miniature brains solve complex tasks. In: Journal of Comparative Physiology A, 189(8), 579-588 (2003).

4. Wehner, R: The desert ant's navigational toolkit: procedural rather than positional knowledge. In: Navigation, 55(2), 101-114 (2008).

5. Labhart, T., Meyer, E. P.: Detectors for polarized skylight in insects: a survey of ommatidial specializations in the dorsal rim area of the compound eye. In: Microscopy research and technique, 47(6), 368-379 (1999).

6. Labhart, T.: Polarization-opponent interneurons in the insect visual system. In: Nature, 331(6155), 435 (1988).

7. Labhart, T., Meyer, E. P.: Detectors for polarized skylight in insects: a survey of ommatidial specializations in the dorsal rim area of the compound eye. In: Microscopy research and technique, 47(6), 368-379 (1999).

8. Wittlinger, M., Wehner, R., Wolf, H.: The desert ant odometer: a stride integrator that accounts for stride length and walking speed. In: Journal of experimental Biology, 210(2), 198-207 (2007).

9. Ronacher, B., Gallizzi, K., Wohlgemuth, S., Wehner, R.: Lateral optic flow does not influence distance estimation in the desert ant Cataglyphis fortis. In Journal of Experimental Biology, 203(7), 1113-1121 (2000). 
10. Lambrinos, D., Kobayashi, H., Pfeifer, R., Maris, M., Labhart, T., Wehner, R: An autonomous agent navigating with a polarized light compass. In: Adaptive behavior, 6(1), 131-161 (1997).

11. Moller, R., Lambrinos, D., Roggendorf, T., Pfeifer, R., Wehner, R.: Insect strategies of visual homing in mobile robots. In: Proceedings of the Computer Vision and Mobile Robotics Workshop CVMR (Vol. 98) (2001).

12. Chu, J., Zhao, K., Zhang, Q., Wang, T.: Construction and performance test of a novel polarization sensor for navigation. In: Sensors and Actuators A: Physical, 148(1), 75-82 (2008).

13. Chu, J. K., Wang, Z. W., Guan, L., Liu, Z., Wang, Y. L., Zhang, R.: Integrated polarization dependent photodetector and its application for polarization navigation. In: IEEE Photonics Technol. Lett, 26(5), 469-472 (2014).

14. Chu, J., Wang, H., Chen, W., Li, R.: Application of a novel polarization sensor to mobile robot navigation. In: Mechatronics and Automation, 2009. ICMA 2009. International Conference on (pp. 3763-3768). IEEE (2009).

15. Stürzl, W., Carey, N.: A fisheye camera system for polarisation detection on UAVs. In: European Conference on Computer Vision (pp. 431-440). Springer, Berlin, Heidelberg, (2012).

16. Stürzl, W.: A Lightweight Single-Camera Polarization Compass with Covariance Estimation. In: IEEE International Conference on Computer Vision. (2017).

17. Dupeyroux, J., Passault, G., Ruffier, F., Viollet, S., Serres, J.: Hexabot: a small 3D-printed six-legged walking robot designed for desert ant-like navigation tasks. In: 20th IFAC Word Congress 2017, Toulouse, France, 16628-16631 (2017).

18. Dupeyroux, J., Diperi, J., Boyron, M., Viollet, S., Serres, J.: A novel insect-inspired optical compass sensor for a hexapod walking robot. In: IROS 2017-IEEE/RSJ International Conference on Intelligent Robots and Systems, Vancouver, Canada, 3439-3445 (2017).

19. Dupeyroux, J., Diperi, J., Boyron, M., Viollet, S., Serres, J.: A bio-inspired celestial compass applied to an ant-inspired robot for autonomous navigation. In: ECMREuropean Conference on Mobile Robotics, Paris, France (2017).

20. Mafrica, S., Godiot, S., Menouni, M., Boyron, M., Expert, F., Juston, R., Marchand, N., Ruffier, F., Viollet, S.: A bio-inspired analog silicon retina with MichaelisMenten auto-adaptive pixels sensitive to small and large changes in light. In: Optics express, 23(5), 5614-5635 (2015).

21. Dupeyroux, J., Boutin, V., Serres, J., Perrinet, L., Viollet, S.: M²APix: a bioinspired auto-adaptive visual sensor for robust ground height estimation. Accepted In: Circuits and Systems (ISCAS), 2018 IEEE International Symposium on, Florence, Italy (2018).

22. Vanhoutte, E., Mafrica, S., Ruffier, F., Bootsma, R. J., Serres, J.: Time-of-travel methods for measuring optical flow on board a micro flying robot. In: Sensors, 17(3), 571 (2017).

23. Haferlach, T., Wessnitzer, J., Mangan, M., Webb, B.: Evolving a neural model of insect path integration. In: Adaptive Behavior, 15(3), 273-287 (2007).

24. Stone, T., Webb, B., Adden, A., Weddig, N. B., Honkanen, A., Templin, R., Wcislo, W., Scimeca, L., Warrant, E., Heinze, S.: An anatomically constrained model for path integration in the bee brain. In: Current Biology, 27(20), 3069-3085 (2017).

25. Lin, P. C., Komsuoglu, H., Koditschek, D. E.: A leg configuration measurement system for full-body pose estimates in a hexapod robot. In: IEEE Transactions on robotics, 21(3), 411-422 (2005). 